Reprod. Nutr. Dévelop., 1988, 28 (1), 181-182.

\title{
Réponses motrices du côlon aux constituants pariétaux et à la finesse de mouture des aliments chez le lapin
}

\author{
T. BOUYSSOU, M. CANDAU $\left({ }^{*}\right)$, Y. RUCKEBUSCH \\ Ecole Nationale Vétérinaire, 31076 Toulouse Cedex, \\ (*) Ecole Nationale Supérieure Agronomique, 31076 Toulouse Cedex.
}

Summary. The characteristics of the large intestine electromyogram of rabbits were defined for two diets, dehydrated lucerne and dehydrated beet pulp, each of them being either coarsely or finely ground before pelleting. Fineness of grinding was a major factor involved in the changes of colonic motor patterns.

La nature des fibres alimentaires et la finesse de mouture d'un aliment sont des facteurs modifiant le transit digestif chez le lapin (Laplace et Lebas, 1977 ; Lebas et Laplace, 1977), une espèce pour laquelle le temps de rétention colique correspond à environ $80 \%$ du temps total de transit. Dans cette étude, sont envisagées les particularités motrices du côlon induites par deux aliments qui diffèrent par la nature des constituants pariétaux et par deux finesses de mouture.

Matériel et méthodes. Deux aliments, I'un de luzerne déshydratée $(40 \%)$, l'autre de pulpe de betterave déshydratée $(50 \%)$, ont été broyés avant granulation (grille de $1 \mathrm{~mm}$ ou de $4 \mathrm{~mm}$; Pairet et al., 1986). Chacun des aliments a été distribué dès le sevrage à un lot de quatre lapins de race Néo-Zélandaise. Un mois après le début des régimes, six groupes d'électrodes intrapariétales ont été implantés (Ruckebusch, 1973) sur le cæcum, le côlon proximal et le côlon distal.

L'activité électrique a été recueillie sur un polygraphe (Reega VIII, Alvar, Paris) de façon continue durant $48 \mathrm{~h}$ chez chacun des sujets, 7 à 15 jours après l'opération. L'électromyogramme est décrit selon deux types d'activité rapide : l'une localisée (AL), l'autre propagée (AP). L'analyse des tracés permet d'établir leur durée respective exprimée en pour cent du temps d'enregistrement ainsi que la proportion d'ondes péristaltiques et antipéristaltiques sur le côlon proximal (Cherbut et Ruckebusch, 1985).

Résultats et discussion. L'activité électrique rapide du côlon par rapport aux variations lentes de potentiels est organisée en salves de potentiels ( 2 et 4 s), sans propagation apparente entre deux autres groupes d'électrodes et en salves $(4$ à 15 s) propagées (10 et $\left.40 \mathrm{~mm} . \mathrm{s}^{-1}\right)$ dans le sens iso- ou anti-péristaltique. La différence détectée en fonction de la nature de l'aliment siège sur la partie moyenne du côlon (Fusus coli) et correspond à une activité électrique accrue de $10 \%$ du temps d'enregistrement dans le cas de l'aliment pulpe. En revanche, la finesse de mouture de l'aliment affecte le profil électromyographique du côlon. Lorsque les aliments sont finement broyés, I'activité électrique occupe 35-40\% du temps d'enregistrement pour l'ensemble du côlon (fig. 1). Cette activité est de 
type localisé (26\% du temps d'enregistrement). Quant à l'activité propagée sur le côlon proximal, elle est surtout antipéristaltique : $59 \%$ des salves $(P<0,05)$. Lorsque la mouture de l'aliment est grossière, l'activité électrique n'occupe que 15 à $20 \%$ du temps d'enregistrement. Cette activité apparaît toutes les 2 à 4 min pendant une durée de $120 \mathrm{~s}$ environ. Elle est essentiellement de type propagé : $15 \%$ du temps d'enregistrement, avec $62 \%$ de salves péristaltiques sur le côlon proximal $(P<0,05)$.

Il apparaît ainsi que la finesse de mouture d'un aliment est un facteur modifiant le profil moteur avec activité localisée et antipéristaltique pour un broyage fin, activité propagée par un aliment grossier.

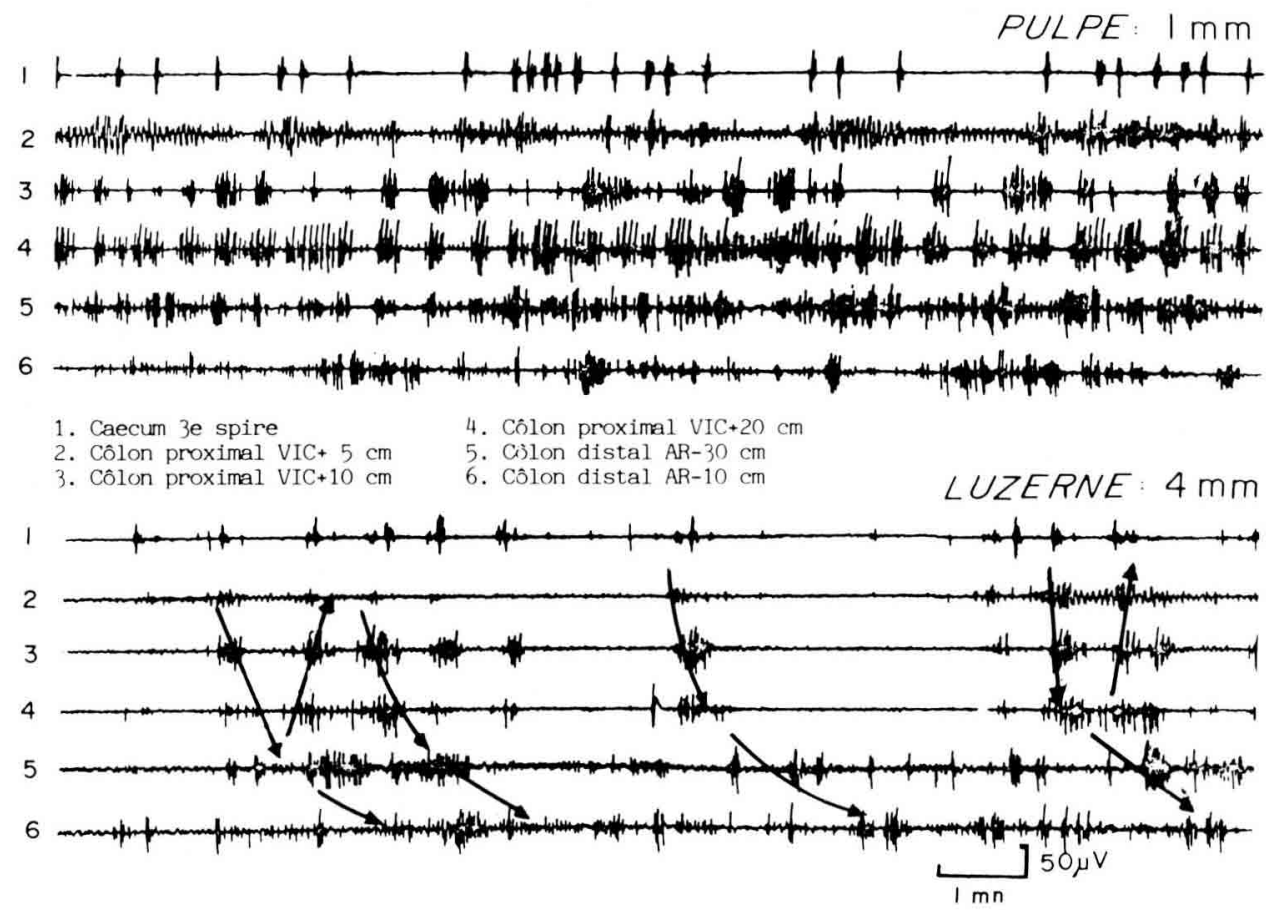

FIG. 1. - Electromyogramme du segment cæco-colique. En haut, activité électrique de type localisé dans le cas d'un aliment pulpe fine mouture. En bas, activité de type propagé dans le cas d'un aliment luzerne mouture grossière. Tracé correspondant à l'élimination de crottes dures.

Cherbut C., Ruckebusch Y., 1985. Br. J. Nutr., 53, 549-557.

Laplace J. P., Lebas F., 1977. Ann. Zootech., 26, 413-420.

Lebas F., Laplace J. P., 1977. Ann. Zootech., 26, 575-584.

Pairet M., Bouyssou T., Auvergne A., Candau M., Ruckebusch Y., 1986. Reprod. Nutr. Dévelop., 26, 85-95.

Ruckebusch Y., 1973, Revue Méd. vét., 124, 1407-1434. 